PROCEEDINGS OF THE

AMERICAN MATHEMATICAL SOCIETY

Volume 135, Number 9, September 2007, Pages 3005-3011

S 0002-9939(07)08783-7

Article electronically published on March 30, 2007

\title{
GEOMETRIC ANGLE STRUCTURES ON TRIANGULATED SURFACES
}

\author{
REN GUO
}

(Communicated by Alexander N. Dranishnikov)

\begin{abstract}
In this paper we characterize the edge invariant and Delaunay invariant of a spherical angle structure on a triangulated surface. We also characterize the edge invariant of a hyperbolic angle structure on a triangulated surface.
\end{abstract}

\section{INTRODUCTION}

Suppose $S$ is a closed surface and $T$ is a triangulation of $S$. Here by a triangulation we mean the following: take a finite collection of triangles and identify their edges in pairs by homeomorphism. Let $V, E, F$ be the sets of all vertices, edges and triangles in $T$ respectively. If $a, b$ are two simplices in triangulation $T$, we use $a<b$ to denote that $a$ is a face of $b$. Let $C(S, T)=\{(e, f) \mid e \in E, f \in F$, such that $e<f\}$ be the set of all corners of the triangulation. An angle structure on a triangulated surface $(S, T)$ assigns each corner of $(S, T)$ a number in $(0, \pi)$. A Euclidean (or hyperbolic, or spherical) angle structure is an angle structure so that each triangle with the angle assignment is Euclidean (or hyperbolic, or spherical). More precisely, a Euclidean angle structure is a map $x: C(S, T) \rightarrow(0, \pi)$ assigning every corner $i$ (for simplicity of notation, we use one letter to denote a corner) a positive number $x_{i}$ such that $x_{i}+x_{j}+x_{k}=\pi$ whenever $i, j, k$ are three corners of a triangle. A hyperbolic angle structure is a map $x: C(S, T) \rightarrow(0, \pi)$ such that $x_{i}+x_{j}+x_{k}<\pi$. A spherical angle structure is a map $x: C(S, T) \rightarrow(0, \pi)$ such that

$$
\left\{\begin{array}{c}
x_{i}+x_{j}+x_{k}>\pi \\
x_{j}+x_{k}-x_{i}<\pi
\end{array}\right.
$$

Actually it is proved in [1] that positive numbers $x_{i}, x_{j}, x_{k}$ are three inner angles of a spherical triangle if and only if they satisfy conditions (1.1).

Given an angle structure $x: C(S, T) \rightarrow(0, \pi)$, its edge invariant is a function $D_{x}: E \rightarrow(0,2 \pi)$ defined by $D_{x}(e)=x_{i}+x_{i^{\prime}}$ where $i=(e, f), i^{\prime}=\left(e, f^{\prime}\right)$ are two opposite corners facing the edge $e$, and its Delaunay invariant is a function $\mathcal{D}_{x}: E \rightarrow(-2 \pi, 2 \pi)$ defined by $\mathcal{D}_{x}(e)=x_{j}+x_{k}+x_{j^{\prime}}+x_{k^{\prime}}-x_{i}-x_{i^{\prime}}$ where $i=(e, f), i^{\prime}=\left(e, f^{\prime}\right)$ are two opposite corners facing the edge $e$ and $j, k$ (or $\left.j^{\prime}, k^{\prime}\right)$ are the other two corners of the triangle $f$ (or $f^{\prime}$ ). The concept of edge invariant

Received by the editors March 30, 2006 and, in revised form, May 9, 2006.

2000 Mathematics Subject Classification. Primary 57M50; Secondary 90C05.

Key words and phrases. Geometric angle structures, linear programming.

(C)2007 American Mathematical Society Reverts to public domain 28 years from publication 
was first introduced by Rivin [5]. The concept of Delaunay invariant was first introduced by Leibon [3].

For simplicity, let $G=E, H$ or $S$ be the Euclidean, hyperbolic or spherical geometry respectively. Given a function $D: E \rightarrow(0,2 \pi)$, the set of all $G$ angle structures with edge invariant $D$ is denoted by $A G(S, T ; D)$. Given a function $\mathcal{D}: E \rightarrow(-2 \pi, 2 \pi)$, the set of all $G$ angle structures with Delaunay invariant $\mathcal{D}$ is denoted by $A G(S, T ; \mathcal{D})$.

The motivation for considering these sets is the study of geometric cone metrics with prescribed edge invariant or Delaunay invariant on triangulated surfaces from the variational point of view. A Euclidean (or hyperbolic, or spherical) cone metric assigns each edge in $T$ a positive number such that the numbers on any three edges of a triangle in $T$ can be realized as three edge lengths of a Euclidean (or hyperbolic, or spherical) triangle. The variational method contains a variational problem and a linear programming problem. The variational problem is to show that the unique maximal point of a convex capacity defined on the set $A G(S, T ; D)$ or $A G(S, T ; \mathcal{D})$ gives the unique geometric cone metric. The linear programming problem is to characterize the function $D$ or $\mathcal{D}$ such that the set $A G(S, T ; D)$ or $A G(S, T ; \mathcal{D})$ is nonempty.

For Euclidean angle structures, the Delaunay invariant and the edge invariant are related by $2 D_{x}(e)+\mathcal{D}_{x}(e)=2 \pi$ for any edge $e$. Thus given two functions $D$ and $\mathcal{D}$ satisfying $2 D(e)+\mathcal{D}(e)=2 \pi$ for any edge $e$, we have $A E(S, T ; D)=A E(S, T ; \mathcal{D})$. Therefore the problem of a Euclidean cone metric with a given edge invariant is equivalent to the problem of a Euclidean cone metric with a given Delaunay invariant. Rivin [5], 6] worked out the variational problem and the linear programming problem for $A E(S, T ; D)$. Leibon [3] worked out the variational problem and the linear programming problem for $A H(S, T ; \mathcal{D})$. Luo 4 ] worked out the variational problem for $A S(S, T ; D)$, the linear programming problem about which will be solved in this paper (Theorem 1.1). Although the variational problems for $A H(S, T ; D)$ and $A S(S, T ; \mathcal{D})$ are still open, we will solve the linear programming problem for them in this paper (Theorem 1.2 and 1.3). The main result of this paper, Theorems 1.1, 1.2 and [1.3, is an extension of Rivin's result [6] and Leibon's result [3. They are stated in the same style as the following.

For a triangulated surface $(S, T)$ and a subset $X \subseteq F$, the number of triangles in $X$ is denoted by $|X|$ and the set of all edges of triangles in $X$ is denoted by $E(X)$.

Theorem 1.1. Given a triangulated surface $(S, T)$ and a function $D: E \rightarrow(0, \pi)$, the set $A S(S, T ; D)$ is nonempty if and only if for any subset $X \subseteq F$,

$$
\pi|X|<\sum_{e \in E(X)} D(e) .
$$

Theorem 1.2. Given a triangulated surface $(S, T)$ and a function $D: E \rightarrow(0,2 \pi)$, the set $A H(S, T ; D)$ is nonempty if and only if for any subset $X \subset F$,

$$
\pi(|F|-|X|)>\sum_{e \notin E(X)} D(e) .
$$

Theorem 1.3. Given a triangulated surface $(S, T)$ and a function $\mathcal{D}: E \rightarrow$ $(-2 \pi, 2 \pi)$, the set $A S(S, T ; \mathcal{D})$ is nonempty if and only if for any subset $X \subset F$,

$$
\pi(|F|-|X|)>\sum_{e \notin E(X)}\left(\pi-\frac{1}{2} \mathcal{D}(e)\right) .
$$


The paper is organized as follows. In section 2, we prove Theorem 1.1 by using Leibon's result. In section 3, we recall the duality theorem in linear programming. In section 4, following Rivin's method, we prove Theorems 1.2 and 1.3 by using the duality theorem.

\section{Proof of Theorem 1.1}

First let us recall Leibon's result for the characterization of the function $\mathcal{D}$ such that the set $A H(S, T ; \mathcal{D})$ is nonempty.

Theorem 2.1 (Leibon [3]). Given a triangulated surface $(S, T)$ and a function $\mathcal{D}: E \rightarrow(0,2 \pi)$, the set $A H(S, T ; \mathcal{D})$ is nonempty if and only if for any subset $X \subseteq F$,

$$
\pi|X|<\sum_{e \in E(X)}\left(\pi-\frac{1}{2} \mathcal{D}(e)\right)
$$

Proof of Theorem 1.1. To show the conditions are necessary, for any $X \subseteq F$, we have $\sum_{e \in E(X)} D(e)=\sum_{e \in E(X)}\left(x_{i}+x_{i^{\prime}}\right)$, where $i, i^{\prime}$ are two opposite corners facing the edge $e$. It turns out that the right-hand side of the equation is equal to $\sum_{f \in X}\left(x_{i}+x_{j}+x_{k}\right)+\sum x_{h}$, where the corner $h=\left(e, f^{*}\right)$ with $e \in E(X)$ and $f^{*} \notin X$. Hence $\sum_{e \in E(X)} D(e) \geq \sum_{f \in X}\left(x_{i}+x_{j}+x_{k}\right)>\sum_{f \in X} \pi=\pi|X|$.

To show the conditions are sufficient, let us define a function $\mathcal{D}: E \rightarrow(0,2 \pi)$ by setting $\mathcal{D}(e)=2 \pi-2 D(e)$. Thus the conditions $\pi|X|<\sum_{e \in E(X)} D(e)$ are equivalent to $\pi|X|<\sum_{e \in E(X)}\left(\pi-\frac{1}{2} \mathcal{D}(e)\right)$, which guarantees that $A H(S, T ; \mathcal{D})$ is nonempty by Theorem 2.1. It follows that there is a solution for the inequalities

$$
\left\{\begin{array}{c}
x_{i}+x_{j}+x_{k}<\pi \\
x_{j}+x_{k}+x_{j^{\prime}}+x_{k^{\prime}}-x_{i}-x_{i^{\prime}}=\mathcal{D}(e) \\
x_{i}>0
\end{array}\right.
$$

Let us define new variables $y_{i}$ for all $i \in C(S, T)$ by setting

$$
y_{i}=\frac{\pi+x_{i}-x_{j}-x_{k}}{2}
$$

provided $i, j, k$ are three corners of a triangle. Since $\mathcal{D}(e)=2 \pi-2 D(e)$, the inequalities above are equivalent to

$$
\left\{\begin{array}{cc}
y_{i}+y_{j}+y_{k}>\pi & i, j, k \text { are three corners of a triangle } \\
y_{i}+y_{i^{\prime}}=D(e) & i, i^{\prime} \text { are two opposite corners facing an edge } e \\
y_{j}+y_{k}<\pi & j, k \text { are two corners of a triangle. }
\end{array}\right.
$$

This solution obviously satisfies

$$
\left\{\begin{array}{cc}
y_{i}+y_{j}+y_{k}>\pi & i, j, k \text { are three corners of a triangle } \\
y_{i}+y_{i^{\prime}}=D(e) & i, i^{\prime} \text { are two opposite corners facing an edge } e \\
y_{j}+y_{k}-y_{i}<\pi & i, j, k \text { are three corners of a triangle. } \\
y_{i}>0 &
\end{array}\right.
$$

Thus we obtain an angle structure in $A S(S, T ; D)$. 


\section{Duality Theorem}

We fix the notation as follows: $x=\left(x_{1}, \ldots, x_{n}\right)^{t}$ is a column vector in $\mathbf{R}^{n}$. The standard inner product in $\mathbf{R}^{n}$ is denoted by $a^{t} x$. If $A: \mathbf{R}^{n} \rightarrow \mathbf{R}^{m}$ is a linear transformation, we denote its transpose by $A^{t}: \mathbf{R}^{m} \rightarrow \mathbf{R}^{n}$. Given two vectors $x, a$ in $\mathbf{R}^{n}$, we say $x \geq a$ if $x_{i} \geq a_{i}$ for all indices $i$. Also $x>a$ means $x_{i}>a_{i}$ for all indices $i$.

A linear programming problem $(P)$ is to minimize an objective function $z=a^{t} x$ subject to the restraint conditions

$$
\left\{\begin{array}{c}
A x=b \\
x \geq 0
\end{array}\right.
$$

where $x \in \mathbf{R}^{n}, b \in \mathbf{R}^{m}$ and $A: \mathbf{R}^{n} \rightarrow \mathbf{R}^{m}$ is a linear transformation. We call a point $x$ satisfying the restraint conditions a feasible solution and denote the set of all the feasible solutions by $D(P)=\left\{x \in \mathbf{R}^{n} \mid A x=b, x \geq 0\right\}$. An optimal solution $x$ for $(P)$ is a feasible solution so that the objective function $z$ realizes the minimal value. The dual problem $\left(P^{*}\right)$ of $(P)$ is to maximize $z=b^{t} y$ subject to $A^{t} y \leq a, y \in \mathbf{R}^{m}$. Let us recall the duality theorem in linear programming. The proof of the theorem can be found in the book [2].

Theorem 3.1. The following statements are equivalent.

(1) Problem $(P)$ has an optimal solution.

(2) $D(P) \neq \emptyset$ and $D\left(P^{*}\right) \neq \emptyset$.

(3) Both problem $(P)$ and problem $\left(P^{*}\right)$ have optimal solutions so that the minimal value of $(P)$ is equal to the maximal value of $\left(P^{*}\right)$.

In applications that we are interested in, there is a special case in which the objective function $z=0$ for $(P)$. Thus the optimal solution exists if and only if $D(P) \neq \emptyset$. Thus we obtain the following corollary.

Corollary 3.2. For $A: \mathbf{R}^{n} \rightarrow \mathbf{R}^{m}$ and $b \in \mathbf{R}^{m}$, the set $\left\{x \in \mathbf{R}^{n} \mid A x=b, x \geq 0\right\} \neq$ $\emptyset$ if and only if the maximal value of $z=b^{t} y$ on $\left\{y \in R^{m} \mid A^{t} y \leq 0\right\}$ is nonpositive.

\section{Proof of Theorems 1.2 and 1.3}

By following Rivin's method in [6], we will prove a lemma about the closure of $A H(S, T ; D)$ in $\mathbf{R}^{3|F|}=\left\{\left(x_{i}\right)^{t}, i \in C(S, T)\right\}$. The closure of $A H(S, T ; D)$ consists of all the points satisfying

$$
\left\{\begin{array}{c}
x_{i}+x_{j}+x_{k} \leq \pi \\
x_{i}+x_{i^{\prime}}=D(e) \\
x_{i} \geq 0
\end{array} \quad i, i^{\prime} \text { are two opposite corners facing an edge } e,\right.
$$

Lemma 4.1. Given a triangulated surface $(S, T)$ and a function $D: E \rightarrow[0,2 \pi]$, the closure of $A H(S, T ; D)$ is nonempty if and only if for any subset $X \subset F$,

$$
\pi(|F|-|X|) \geq \sum_{e \notin E(X)} D(e) .
$$

Proof. The linear programming problem $(P)$ with variables $x=\left(\ldots, x_{i}, \ldots, t_{f}, \ldots\right)$ indexed by $C(S, T) \cup F$ is to minimize the objective function $z=0$ subject to the 
restraint conditions

$$
\left\{\begin{array}{c}
x_{i}+x_{j}+x_{k}+t_{f}=\pi \quad i, j, k \text { are three corners of a triangle } f \\
x_{i}+x_{i^{\prime}}=D(e) \\
x_{i} \geq 0 \\
t_{f} \geq 0
\end{array} \quad i, i^{\prime} \text { are two opposite corners facing an edge } e,\right.
$$

The dual problem $\left(P^{*}\right)$ with variable $y=\left(\ldots, y_{f}, \ldots, y_{e}, \ldots\right)$ indexed by $E \cup F$ is to maximize the objective function $z=\sum_{f \in F} \pi y_{f}+\sum_{e \in E} D(e) y_{e}$ subject to the restraint conditions

$$
\left\{\begin{array}{c}
y_{f} \leq 0 \\
y_{f}+y_{e} \leq 0 \quad \text { whenever } e<f
\end{array}\right.
$$

Since the closure of $A H(S, T ; D)$ is nonempty is equivalent to that the set $D(P)$ is nonempty, by Corollary 3.2 , the latter one is equivalent to that the maximal value of the objective function of $\left(P^{*}\right)$ is nonpositive.

To show the conditions $\pi(|F|-|X|) \geq \sum_{e \notin E(X)} D(e)$ for any $X \subset F$ are necessary, for any $X \subset F$, let

$$
y_{f}=\left\{\begin{array}{cl}
0 & \text { if } f \in X \\
-1 & \text { if } f \notin X
\end{array} \quad \text { and } y_{e}=\left\{\begin{array}{cc}
0 & \text { if } e \in E(X) \\
1 & \text { if } e \notin E(X) .
\end{array}\right.\right.
$$

We claim that $\left(y_{f}, y_{e}\right)$ is a feasible solution. In fact, given a pair $e<f$, if $f \in X$, we must have $e \in E(X)$, then $y_{f}+y_{e}=0$. If $f \notin X$, then $y_{f}+y_{e}=-1+y_{e} \leq 0$.

By the assumption that the maximal value of the objective function of $\left(P^{*}\right)$ is nonpositive, since $\left(y_{f}, y_{e}\right)$ is feasible, we have $0 \geq z\left(y_{f}, y_{e}\right)=\sum_{f \notin X} \pi y_{f}+$ $\sum_{e \notin E(X)} D(e) y_{e}=\pi(|X|-|F|)+\sum_{e \notin E(X)} D(e)$.

To show the conditions are sufficient, take an arbitrary feasible solution $\left(y_{f}, y_{e}\right)$. If $y_{f}=0$ for all $f$, from $y_{f}+y_{e} \leq 0$, we know $y_{e} \leq 0$. Hence $z\left(y_{f}, y_{e}\right)=$ $\sum_{e \notin E} D(e) y_{e} \leq 0$, since $D(e) \in[0,2 \pi]$. Otherwise, define $X=\left\{f \in F \mid y_{f}=\right.$ $0\} \subset F$, and let $a=\max \left\{y_{f}, f \notin X\right\}$. We have $a<0$. Define

$$
y_{f}^{(1)}=\left\{\begin{array}{ll}
y_{f}=0 & \text { if } f \in X \\
y_{f}-a & \text { if } f \notin X
\end{array} \text { and } y_{e}^{(1)}=\left\{\begin{array}{cl}
y_{e} & \text { if } e \in E(X) \\
y_{e}+a & \text { if } e \notin E(X) .
\end{array}\right.\right.
$$

We claim that $\left(y_{f}^{(1)}, y_{e}^{(1)}\right)$ is a feasible solution. In fact, $y_{f}^{(1)} \leq 0$. Given a pair $e<f$, if $f \in X$, we must have $e \in E(X)$. Then $y_{f}^{(1)}+y_{e}^{(1)}=y_{f}+y_{e} \leq 0$. If $f \notin X$ and $e \notin E(X)$, then $y_{f}^{(1)}+y_{e}^{(1)}=y_{f}-a+y_{e}+a \leq 0$. If $f \notin X$ but $e \in E(X)$, there exists another triangle $f^{\prime} \in X$ so that $e<f^{\prime}$. Then $y_{e}=y_{e}+y_{f^{\prime}} \leq 0$. Therefore $y_{f}^{(1)}+y_{e}^{(1)}=y_{f}-a+y_{e} \leq y_{f}-a \leq 0$, since $a$ is the maximum.

Now the value of the objective function is $z\left(y_{f}^{(1)}, y_{e}^{(1)}\right)=z\left(y_{f}, y_{e}\right)+a(\pi(|X|-$ $\left.|F|)+\sum_{e \notin E(X)} D(e)\right) \geq z\left(y_{f}, y_{e}\right)$, according to the conditions. Note the number of 0 's in $\left\{y_{f}^{(1)}\right\}$ is more than that in $\left\{y_{f}\right\}$. By the same procedure, after a finite number of steps, it ends at a feasible solution $\left(y_{f}^{(n)}=0, y_{e}^{(n)}\right)$. We have $z\left(y_{f}^{(n)}, y_{e}^{(n)}\right) \leq 0$. Since the value of the objective function does not increase, therefore $0 \geq z\left(y_{f}^{(n)}, y_{e}^{(n)}\right) \geq \ldots \geq z\left(y_{f}^{(1)}, y_{e}^{(1)}\right) \geq z\left(y_{f}, y_{e}\right)$.

Proof of Theorem 1.2. Let $x_{i}=a_{i}+\varepsilon$ for any $i \in C(S, T)$, where $a_{i} \geq 0$ and $\varepsilon \geq 0$. The linear programming problem $(P)$ with variables $\left\{\ldots, a_{i}, \ldots \varepsilon\right\}$ is to minimize the 
objective function $z=-\varepsilon$ subject to the restraint conditions

$$
\left\{\begin{array}{c}
a_{i}+a_{j}+a_{k}+3 \varepsilon \leq \pi \quad i, j, k \text { are three corners of a triangle, } \\
a_{i}+a_{j}+2 \varepsilon=D(e) \quad i, j \text { are two opposite corners facing an edge } e, \\
a_{i} \geq 0 \\
\varepsilon \geq 0
\end{array}\right.
$$

The dual problem $\left(P^{*}\right)$ with variable $y=\left(\ldots, y_{f}, \ldots, y_{e}, \ldots\right)$ indexed by $E \cup F$ is to maximize the objective function $z=\sum_{f \in F} \pi y_{f}+\sum_{e \in E} D(e) y_{e}$ subject to the restraint conditions

$$
\left\{\begin{array}{c}
y_{f} \leq 0 \\
y_{f}+y_{e} \leq 0 \\
3 \sum_{f \in F} y_{f}+2 \sum_{e \in E} y_{e} \leq-1 .
\end{array} \quad \text { whenever } e<f\right.
$$

By Theorem 3.1(3), the maximal value of the objective function of $\left(P^{*}\right)$ is negative is equivalent to that the minimal value of the objective function of $(P)$ is negative. The latter one is equivalent to that there exists a feasible solution $a_{i} \geq 0, \varepsilon>0$. Therefore the set $A H(S, T ; D)$ is nonempty.

We only need to show that the maximal value of the objective function of $\left(P^{*}\right)$ is negative is equivalent to the conditions $\pi(|F|-|X|)>\sum_{e \notin E(X)} D(e)$ for any $X \subset F$.

To show the conditions are necessary, for any $X \subset F$, we have $2|E(X)|>3|X|$ or $2|E(X)| \geq 3|X|+1$. Let

$$
y_{f}=\left\{\begin{array}{cc}
0 & \text { if } f \in X \\
-1 & \text { if } f \notin X
\end{array} \text { and } y_{e}=\left\{\begin{array}{cc}
0 & \text { if } e \in E(X) \\
1 & \text { if } e \notin E(X) .
\end{array}\right.\right.
$$

We claim that $\left(y_{f}, y_{e}\right)$ is a feasible solution. If fact, as in Lemma 4.1, we can check $y_{f}+y_{e} \leq 0$ for any pair $e<f$. Furthermore

$$
\begin{gathered}
3 \sum_{f \in F} y_{f}+2 \sum_{e \in E} y_{e}=3 \sum_{f \notin X}(-1)+2 \sum_{e \notin E(X)} 1=3(|X|-|F|)+2(|E|-|E(X)|) \\
=3|X|-2|E(X)|+2|E|-3|F|=3|X|-2|E(X)| \leq-1
\end{gathered}
$$

since $2|E|=3|F|$. Now $\left(y_{f}, y_{e}\right)$ is feasible implies that $z\left(y_{f}, y_{e}\right)<0$, which is equivalent to $\pi(|F|-|X|)<\sum_{e \notin E(X)} D(e)$.

To show the conditions are sufficient, by the proof of Lemma 4.1 we know that the maximal value of the objective function of $\left(P^{*}\right)$ is $\leq 0$ under the conditions. We try to show it cannot be 0 . Assume that $\left(y_{f}, y_{e}\right)$ is a feasible solution satisfying $z\left(y_{f}, y_{e}\right)=0$. We claim that $y_{f}=0$ for all $f$. Otherwise, as in the proof of Lemma 4.1, we can find another feasible solution $\left(y_{f}^{(1)}, y_{e}^{(1)}\right)$ and we can check that $z\left(y_{f}^{(1)}, y_{e}^{(1)}\right)=z\left(y_{f}, y_{e}\right)+a\left(\pi(|X|-|F|)+\sum_{e \notin E(X)} D(e)\right)>z\left(y_{f}, y_{e}\right)=0$, according to the conditions. It is a contradiction since the maximal value of the objective function of $\left(P^{*}\right)$ is $\leq 0$.

Now from $y_{f}=0$ for all $f$ we see $y_{e} \leq 0$. Since $0=z\left(y_{f}, y_{e}\right)=\sum_{e \in E} D(e) y_{e}$ and $D(e)>0$, we get $y_{e}=0$ for all $e$ and therefore $\left(y_{f}, y_{e}\right)=(0,0)$. But $\left(y_{f}, y_{e}\right)=(0,0)$ does not satisfy $3 \sum_{f \in F} y_{f}+2 \sum_{e \in E} y_{e} \leq-1$. It is a contradiction since we assume that $\left(y_{f}, y_{e}\right)$ is a feasible solution. This proves that the maximal value of the objective function of $\left(P^{*}\right)$ is negative.

Proof of Theorem 1.3. Given two functions $D: E \rightarrow(0,2 \pi)$ and $\mathcal{D}: E \rightarrow(-2 \pi, 2 \pi)$ satisfying $2 D(e)+\mathcal{D}(e)=2 \pi$ for any $e$, we claim that $A H(S, T ; D) \neq \emptyset$ is equivalent 
to $A S(S, T ; \mathcal{D}) \neq \emptyset$. By this claim, Theorem 1.3 is true as a corollary of Theorem 1.2 .

In fact, $A S(S, T ; \mathcal{D})$ is the set of solutions for the inequalities

$$
\left\{\begin{array}{cl}
x_{i}+x_{j}+x_{k}>\pi & i, j, k \text { are three corners of a triangle } \\
x_{j}+x_{k}-x_{i}<\pi & i, j, k \text { are three corners of a triangle } \\
x_{j}+x_{k}+x_{j^{\prime}}+x_{k^{\prime}}-x_{i}-x_{i^{\prime}}=\mathcal{D}(e), & \\
x_{i}>0 &
\end{array}\right.
$$

Let us define new variables $y_{i}$ for all $i \in C(S, T)$ by setting

$$
y_{i}=\frac{\pi+x_{i}-x_{j}-x_{k}}{2}
$$

provided $i, j, k$ are three corners of a triangle. Since $2 D(e)+\mathcal{D}(e)=2 \pi$, we see that the inequalities above are equivalent to

$$
\left\{\begin{array}{cc}
y_{i}+y_{j}+y_{k}<\pi & i, j, k \text { are three corners of a triangle } \\
y_{i}>0, & \\
y_{i}+y_{i^{\prime}}=D(e) & i, i^{\prime} \text { are two opposite corners facing an edge } e \\
y_{j}+y_{k}<\pi & j, k \text { are two corners of a triangle. }
\end{array}\right.
$$

Since $y_{i}+y_{j}+y_{k}<\pi$ implies $y_{j}+y_{k}<\pi$, we can omit the latter one. Equivalently, we get

$$
\left\{\begin{array}{c}
y_{i}+y_{j}+y_{k}<\pi \quad i, j, k \text { are three corners of a triangle } \\
y_{i}>0, \\
y_{i}+y_{i^{\prime}}=D(e) \quad i, i^{\prime} \text { are two opposite corners facing an edge } e .
\end{array}\right.
$$

Now the set of solutions of the inequalities above is exactly $A H(S, T ; D)$. Thus we see that $A H(S, T ; D) \neq \emptyset$ is equivalent to $A S(S, T ; \mathcal{D}) \neq \emptyset$.

\section{ACKNOWLEDGMENTS}

I wish to thank my advisor, Professor Feng Luo, for suggesting this problem and for fruitful discussions. I appreciate the referee's helpful advice.

\section{REFERENCES}

1. Marcel Berger, Geometry II. Springer-Verlag 1987 MR0882916 (88a:51001b)

2. Bernard Kolman and Robert Beck, Elementary Linear Programming with Applications. Academic Press, 2nd edition, 1995 MR 1340426 (96f:90001)

3. Gregory Leibon, Characterizing the Delaunay decompositions of compact hyperbolic surfaces. Geom. Topol. 6(2002), 361-391 MR1914573 (2003c:52034)

4. Feng Luo, A Characterization of spherical polyhedron surfaces. ArXiv: math.GT/0408112

5. Igor Rivin, Euclidean structures on simplicial surfaces and hyperbolic volume. Ann. of Math. (2) 139 (1994), no. 3, 553-580 MR1283870 (96h:57010)

6. Igor Rivin, Combinational optimization in geometry. Advances in Applied Math. 31(2003), no. 1, 242-271 MR1985831(2004i:52005)

Department of Mathematics, Rutgers University, Piscataway, New Jersey 08854

E-mail address: renguo@math.rutgers.edu 